\title{
Study of the Changes in Choriodal Thickness Before and after Intravitreal Injection of Ranibizumab in Diabetic Macular Edema
}

\section{Mohamed Abd Elmoamen Mohamed Saad Eldeen ${ }^{1}$ M.Sc; Magdy Ezzat Khallaf ${ }^{1}$ MD; Mostafa Mahmoud Mostafa $^{1}$ MD}

* Corresponding Author:

Mohamed Abd Elmoamen Mohamed Saad Eldeen

mohamadabdelmoamen@gmail.com

Received for publication August 13, 2020; Accepted November 2, 2020; Published online November 2, 2020.

Copyright 2020 The Authors published by Al-Azhar University, Faculty of Medicine, Cairo, Egypt. All rights reserved. This an openaccess article distributed under the legal terms, where it is permissible to download and share the work provided it is properly cited. The work cannot be changed in any way or used commercially.

doi: 10.21608/aimj.2020.37551.1290

${ }^{1}$ Department of Ophthalmology, Faculty of Medicine, Al-Azhar University

\begin{abstract}
Background: Diabetic Macular Edema is a critical complication of diabetic eye disease, the effect of Anti-VEGF injection in diabetic eyes does not stop at the retina alone as it also influences the choroidal circulation.
\end{abstract}

Aim of the study: To compare subfoveal choroidal thickness (SFCT) before and after intravitreal injection of ranibizumab in DME with the use of Cirrus HD-OCT by enhanced depth spectral-domain imaging (EDI-OCT).

Patient and methods: A prospective study that was demonstrated on 50 eyes of 35 cases with DME. Fundus fluorescein angiography was done for all patients. All patients were received three intravitreal injection of $(0.5 \mathrm{mg} / 0.05 \mathrm{ml}$ ranibizumab) with one-month interval. EDI-OCT was done preoperative and one-month following the third injection.

Results: The mean CT (Choroidal Thickness) was $257.62 \pm 49.207 \mu \mathrm{m}$ with a range from $154 \mu \mathrm{m}$ to $361 \mu \mathrm{m}$ preoperatively. While the mean CT was $239.78 \pm 47.225 \mu \mathrm{m}$ with a range from $150 \mu \mathrm{m}$ to $346 \mu \mathrm{m}$ postoperatively, There were obvious changes between the preoperative and the postoperative SFCT measured by EDI-OCT and there was a positive significant correlation among the baseline SFCT and the change of SFCT (p. value 0.03).

Conclusion: Intravitreal injection of $0.5 \mathrm{mg} / 0.05 \mathrm{ml}$ ranibizumab in the treatment of DME resulting in significant reduction of SFCT with greater response in cases with higher baseline SFCT, but improvement of BCVA was related to retinal thickness not to CT which needs further evaluation.

Keywords: Choriodal thickness; Ranibizumab; Diabetic; macular edema

Disclosure: The authors have no financial interest to declare in relation to the content of this article. The Article Processing Charge was paid for by the authors.

Authorship: All authors have a substantial contribution to the article.

\section{INTRODUCTION}

Diabetic macular edema (DME) is a critical eye conditions resulted mainly from hyperglycemia. It is considered a common cause of loss of vision and blindness in diabetics. ${ }^{1}$

There is a complex pathological process with many contributing factors that are responsible for the pathogenesis of DME. ${ }^{2}$

The normal choroid has fundamental rule for retinal function. Until lately, the choroid can be assessed only via laser flowmetry, indocyanine green angiography and ultrasonography. However, these procedures can only show choroidal vessel abnormalities and variations in blood flow; they cannot display the 3D anatomy of choroid layers or the retinal pigment epithelium. ${ }^{3}$
OCT (Optical coherence tomography) is a noninvasive imaging technique, that was used to obtain a high definition segments of retina. Lately, EDI (enhanced depth imaging) spectral-domain OCT has been explained. (EDI) program routinely captures a cross sectional picture with the choroid near the zero delay line to enhance sensitivity on the external edge of the choroid. ${ }^{3}$

The pathogenesis of DME was referred to retinal vascular hyper permeability, which is accompanied by focal leak from micro aneurysms or spread leak from inefficient capillaries when displayed on fluorescein angiography. ${ }^{4}$

Functional imaging findings also presented a decrease in choroidal flow of the blood in eyes with diabetic retinopathy. Till now, the function of 
choroidal perfusion in the path physiology of DME still not clear. ${ }^{5}$

The current purpose of (EDI-OCT), which employing the enhanced depth of field from the inverted picture by locating the OCT device near to the eye, has helped the researchers to study the anatomic variations in the choroid in diabetic eyes. ${ }^{6}$

In this study, we compare SFCT preoperative and postoperative intravitreal injection of ranibizumab in DME using the Cirrus HD-OCT by enhanced depth spectral-domain imaging (EDI-OCT).

\section{PATIENT AND MATERIALS}

The current work is a prospective study that was demonstrated on 50 eyes of 35cases suffering from DME. These patients were received three intravitreal injection of ranibizumab with one month interval. EDI-OCT was done preoperative and a month after the third injection.

Exclusion criteria: were patients who previously had laser photocoagulation, patients with repaired retinal detachment, patients with atrophic macular scars, high myopes , patients with posterior staphyloma, patients who underwent previous vitrectomy, patients with ischemic maculopathy,Patients with tractional DME and patients with previous intravitreal injection.

Preoperative workup: Detailed history taking, full ophthalmological examination with emphasis on best corrected visual acuity (BCVA), biomicroscopic check of for every patient with examination of the iris for neovascularization and examination of the lens for the presence of cataract or pseudophakia, refraction and detailed fundus examination.

Fluorescein Angiography (FA): was done with exclusion of patients with ischemic maculopathy.

OCT: was done excluding patients with tractional macular edema and included EDI-OCT .OCT image acquired then the SFCT(within $100 \mathrm{M}$ from the fovea) was measured manually using the manual measuring ruler.

The procedure: was performed using topical anesthesia as $0.5 \mathrm{mg} / 0.05 \mathrm{ml}$ ranibizumab is injected $4 \mathrm{~mm}$ (3.5 mm in Pseudophakia) from the limbus intravitreally (in the lower temporal quadrant) by a needle (27gauge). Paracentesis was carried out in some patients.

Postoperative examination: Every patient was examined postoperatively to assess his condition and exclude any complications, the intra ocular pressure (IOP) was measured and the patient was assured.

Follow up: All the cases were followedup after 1 and 4 weaks and the postoperative examination was included full ophthalmological examination with special emphasis on BCVA, biomicroscopic assesment of the anterior segment to exclude any changes or complications, fundus examination to detect fundus changes after the injection and EDIOCT (one month after the third injection). Variations in the CT were estimated by subtracting the preoperative from the postoperative CT for eachpatient.

Ethical consideration: A clear printed approval was taken from each patient before being included in the study.

Statistical analysis: Data were investigated and analyzed utelizing the SPSS V-19 (improved by IBM). Quantitative values were reportedin the form of mean $\pm \mathrm{SD}$ (standard deviation).Qualitative data were reportedin the form of numbers and percentage.

\section{RESULTS}

The current study was demonstrated on 50 eyes of 35 cases from both sexes, undergoing intravitreal injection of ranibizumab for DME.

As regard to the demographic data, the mean age of the studied cases was $63.3 \pm 8.963$ yrs. with a ranging from 42 - 85years. The studied cases included 15 patient (30\%) who were males and 35 cases (70 \%) who were females.

This study included 25 patients with diseased right eyes (50\%) and 25 patients with diseased left eyes (50\%).

According to the time interval of the diabetes, this study reported that the mean duration of diabetes in the study population was19.05 \pm 7.327 years with a range from 5 years to 30 years. This study contained 12 cases with diabetes duration from 5 to 10 years (24\%), 22 cases from 11 to 20 years (44\%) and 16 cases from 21 to 30 years (32\%).

This study included 8 cases with normal lens (16\%), 25 cases with Cataractous lens (50\%) and 17 cases with Pseudophakia (34\%) according to the lens status.

Regarding the pre-operative and postoperative BCVA: The mean pre-operative Snellen's BCVA $(0.4 \pm 0.2$ with a range from 0.03 to 0.9 )and the mean post-operative Snellen's BCVA ( $0.5 \pm 0.2$ with a range from 0.05 to 1.0).table (1).

According to the change in BCVA there were 26 cases with improved postoperative BCVA (52\%), 15 cases with the same BCVA postoperative (30\%) and 9 cases with worse BCVA postoperative (18\%). 
As regard preoperative CT was measured using EDIOCT and the mean subfoveal thickness was $257.62 \pm$ $49.207 \mu \mathrm{m}$ with a range from $154 \mu \mathrm{m}$ to $361 \mu \mathrm{m}$ and postoperative CT was measured using EDI-OCT and the mean subfoveal thickness was $239.78 \pm 47.225$ $\mu \mathrm{m}$ with a range from $150 \mu \mathrm{m}$ to $346 \mu \mathrm{m}$ figure (1) \& table( 2)

Regarding the correlation among baseline of SFCT and its change after Anti-VEGF treatment, there was a positive significant correlation among the baseline SFCT and the change of SFCT indicating that higher baseline SFCT is usually associated with greater response after Anti-VEGF therapy figure (2) \& table (3)
As regard the retinal thickness: Preoperative retinal thickness was assessed, and the mean foveal thickness was $455.84 \pm 127.29 \mu \mathrm{m}$ and ranging from $267 \mu \mathrm{m}$ to $773 \mu \mathrm{m}$. Three months postoperative retinal thickness was estimated, and the mean foveal thickness was $384.44 \pm 111.887 \mu \mathrm{m}$ with a range from $229 \mu \mathrm{m}$ to $648 \mu \mathrm{m}$ table (4).

Studying the effect of the change in the retinal thickness on the change of the CT established that there was a positive correlation, but it was statistically insignificant table (5).

The Correlation among the postoperative CT and the postoperative BCVA among the investigated patients proved that there was a negative insignificant correlation ( $p=0.795)$ figure (3) \& table (6)>

Studying the effect of the postoperative retinal thickness on the postoperative BCVA showed statistically significant negative correlation figure (4) \& table (7).
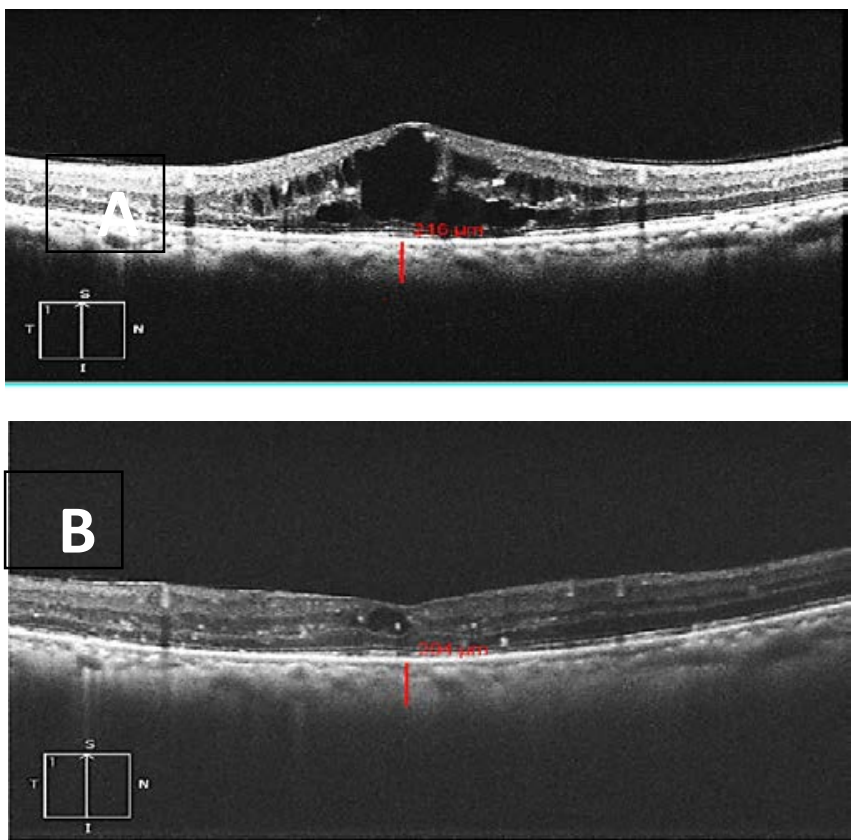

Figure (1): (A)preoperative (B)Postoperative Greyscale EDI-OCT images with CT measurement.

(measured by micrometer) 


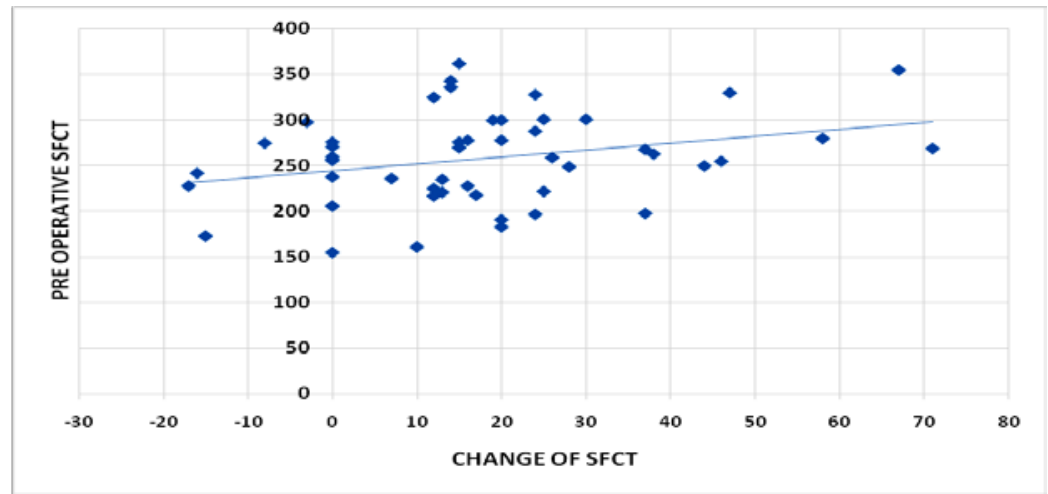

Fig.2: Correlation between the pre-operative CT and the change in CT among the investigated patients.

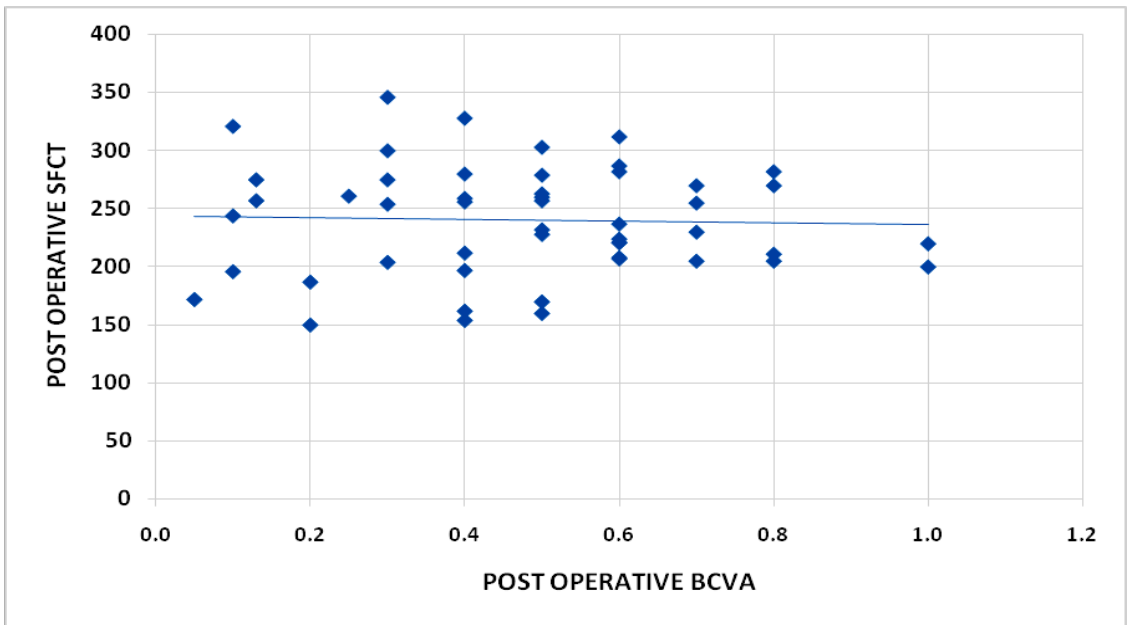

Fig 3: Correlation between the post-operative CT and the post-operative BCVA among the investigated patients. (SFCT: sub foveal choriodal thickness (measured by micrometer) BCVA: best corrected visual acuity)

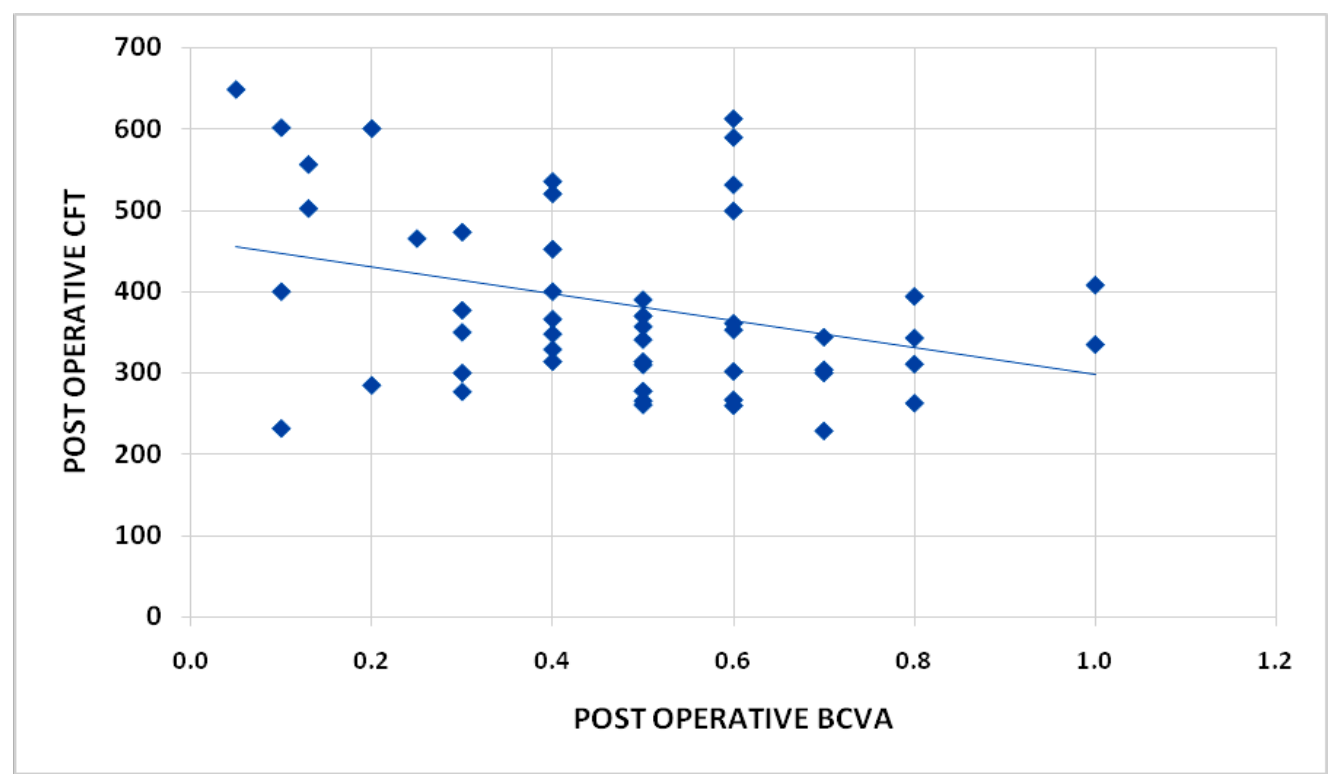

Fig 4: Correlation between the postoperative retinal thickness and the post operative BCVA among the investigated patients. (BCVA: best corrected visual acuity - CFT: central foveal thickness (measured by micrometer)) 


\begin{tabular}{|ccc|}
\hline \multicolumn{1}{|c|}{ Preoperative BCVA } & 3 months postoperative BCVA \\
\hline Range & $0.03-0.9$ & $0.05-1.0$ \\
\hline Mean \pm SD & $0.4 \pm 0.2$ & $0.5 \pm 0.2$ \\
\hline P & 0.001 & \\
\hline
\end{tabular}

Table 1: Mean and SD of the preoperative \& 3 months postoperative BCVA. (BCVA: Best corrected visual acuity - SD standard deviation)

\begin{tabular}{|cccc|}
\hline Choroidal thickness & Preoperative & 3 months postoperative & $\mathrm{P}$ \\
\hline Range & $154-361 \mu \mathrm{m}$ & $150-346 \mu \mathrm{m}$ & $\mathrm{p}<0.001$ \\
\hline Mean \pm SD & $257.62 \pm 49.207 \mu \mathrm{m}$ & $239.78 \pm 47.225 \mu \mathrm{m}$ & \\
\hline
\end{tabular}

Table 2: The mean and SD of pre-operative \& three months post-operative CT. ( $\mu \mathrm{m}$ : micrometer- SD standard deviation)

\begin{tabular}{|c|c|c|} 
& \multicolumn{2}{c|}{ Baseline SFCT } \\
\hline Change of SFCT & $\mathrm{R}$ & $\mathrm{P}$ \\
\hline & 0.297 & 0.036 \\
\hline
\end{tabular}

Table 3: shows positive statistically significant correlation among baseline SFCT and degree of change in it after Anti-VEGF treatment. (SFCT: sub foveal choroidal thickness)

\begin{tabular}{|cccc|}
\hline Retinal foveal thickness & Preoperative & three months postoperative & $\mathrm{P}$ \\
Range & $267-773 \mu \mathrm{m}$ & $229-648 \mu \mathrm{m}$ & $\mathrm{p}<0.001$ \\
\hline Mean \pm SD & $455.84 \pm 127.29 \mu \mathrm{m}$ & $384.44 \pm 111.887 \mu \mathrm{m}$ & \\
\hline
\end{tabular}

Table 4: The mean and standard deviation of preoperative and three months postoperative retinal foveal thickness. ( $\mu \mathrm{m}$ : micrometer)

\begin{tabular}{|lcc|}
\hline \multirow{2}{*}{ Difference in choroidal thickness } & & Difference in retinal thickness \\
\cline { 2 - 3 } & $\mathrm{R}$ & 0.223 \\
\hline & $\mathrm{P}$ & 0.120 \\
\hline
\end{tabular}

Table 5: Correlation among the difference in CT and the difference in retinal thickness between the investigated patients. 


\begin{tabular}{|lcc|}
\hline \multirow{2}{*}{ The postoperative CT } & \multicolumn{3}{c}{ BCVA } \\
\cline { 2 - 3 } & $\mathrm{R}$ & -0.038 \\
\hline $\mathrm{P}$ & 0.795 \\
\hline
\end{tabular}

Table 6: Correlation among the post-operative CT and the post-operative BCVA between the investigated patients. (CT: choriodal thickness - BCVA: Best corrected visual acuity)

\begin{tabular}{|lcc|}
\hline \multirow{2}{*}{ The postoperative retinal thickness } & \multicolumn{2}{c|}{ BCVA } \\
\cline { 2 - 3 } & $\mathrm{R}$ & --0.338 \\
\hline $\mathrm{p}$ & 0.016 \\
\hline
\end{tabular}

Table 7: Correlation among the post-operative retinal thickness and the post-operative BCVA between the investigated patients. (BCVA: Best corrected visual acuity)

\section{DISCUSSION}

Several choroidal anomalies containing obstacle of the choriocapillaries, vascular collapse, choroidal aneurysms and choroidal neovascularization have been described in histopathological studies of diabetic eyes, these changes can be assessed indirectly using CT as an indicator. ${ }^{7}$

Intravitreal injection of Anti-VEGF is an effective and safe treatment to DME which is one of the biggest complications of diabetic eye diseases, the outcome of Anti-VEGF injection in diabetic eyes doesn't stop at the retina alone as it also has an effect on the choroidal circulation. ${ }^{8}$

The CT can be accessed via optical coherence tomography but acceptable visualization of the choroid using (OCT) wasn't available till lately, due to its location and the existence of pigmented cells which weaken the incident light, Spaide et al, developed an Enhanced depth imaging OCT that was a new method which allow a well imagining of the choroid permitting accurate evaluation of the CT. ${ }^{9}$

Latest studies exhibit an efficient evaluation of CT in healthy ${ }^{10}$ and pathological states ${ }^{11}$ utilizing the Cirrus HD-OCT (Carl Zeiss Meditec Inc. Dublin, CA) by Enhanced depth spectral-domain imaging using the Cirrus linear measurement tool, observers measured CT perpendicularly from the external edge of the hyper-reflective retinal pigment epithelium (RPE) to the internal sclera, the central subfoveal thickness was the measure used in almost all the studies to standardize the anatomical factor and avoid anatomical variations. 12

Many researchers have been concerned about studying the choroidal changes in diabetic eye diseases using CT as a reference; though, the effect of Anti-VEGF injection as treatment for DME on the choroidal circulation is an additional issue that needs to be studied carefully, our study aims at studying the effect of ranibizumab on CT. ${ }^{13}$

Spaide et al. concluded that EDI is the finest technique to monitor all the choroid not only the external segments and so it is the best existing technique for examination and follow-up the CT. ${ }^{14}$

In our study the EDI- technique was employed to evaluate the CT. We stressed on the central SFCTto eliminate the anatomical variation and we measured the choroid using the same guidelines from previous studies i.e. vertically from the external edge of the hyper-reflective RPE(retinal pigment epithelium) to the internal sclera. ${ }^{15,16,17}$

We found significant changes between the preoperative and the postoperative measures and the results were as follow: The preoperative SFCTas assessed using EDI-OCT was 257.62 $\pm 49.207 \mu \mathrm{m}$ and ranging from $154 \mu \mathrm{m}$ to $361 \mu \mathrm{m}$. Studies done on the CT of healthy individuals found that it's true value is significantly changed among eyes especially in different age groups.

Erkul et al, in a recent work utilizing EDI-OCT to assay theSFCT reported that the estimated value range is $280.23 \pm 81.15 \mu \mathrm{m}$ in normal adults. ${ }^{18}$

In our study at the postoperative period 4weeksafterward the $3^{\text {rd }}$ injection, the mean subfoveal CT as evaluated using EDI-OCT $239.98 \pm 47.225 \mu \mathrm{m}$ and ranging from $150 \mu \mathrm{m}$ to $346 \mu \mathrm{m}$. The postoperative CT was calculated to be significant with $(\mathrm{p}<$ 0.001) lesser than the pre-operative CT by a mean difference of $17.84 \mu \mathrm{m}$.

From the studies done in post Anti-VEGF injection in diabetic patients; Park YU et al. ${ }^{19}$ compared CT 
previously and afterwards injection of Anti-VEGF using EDI-OCT in 15 eyes and as a part of his consequences he found that: The postoperative CT was reduced after injection of Anti-VEGF with a mean difference of $19 \mu \mathrm{m}$.

The findings of our study compare favourably with this study and with Lee et al. ${ }^{20}$ who reported after studying the effect of intravitreal injection of AntiVEGF on 31 eyes that CT was reduced postoperatively. Another new study done by Lains et al. ${ }^{21}$ on 50 eyes using EDI-OCT also supported the same finding that Diabetic eyes handled with antiVEGF agents have decreased the CT.

Similarly, there was a positive significant correlation among the baseline SFCT and the change of SFCT indicating that higher baseline SFCT is usually associated with greater response after Anti-VEGF therapy (p. value 0.036 ).

In agreement with that, Nadim Rayess et $\mathrm{al}^{22}$ reported that cases with a higher baseline subfoveal CT may suffer from a more intact choriocapillaries and thus a less ischemic external retina, preserving the role of the photoreceptor layer to a wider spread than in cases suffering from a thinner choroid and this may indicate the reason of having a better anatomical response after Anti-VEGF treatment.

Another clarification extracted from another investigation made by Kim et al ${ }^{23}$ reporting that a more thick choroid in DME eyes may be a symptomatic of a more VEGF-driven procedure, that can describe the improved reaction noticed in the present work to anti-VEGF treatment, while eyes with thinner choroids may have DME due to (at least in part) a non-VEGF mediator and are consequently fewer receptive to anti-VEGF treatment.

In the current work the mean preoperative BCVA on snellen's was $(0.4 \pm 0.2)$ while the mean postoperative BVCA on snellen's chart was $(0.5 \pm 0.2)$ with ( $p$ $=0.001$ ).

There were 26 cases with improved postoperative BCVA (52\%), 15 cases with the same BCVA postoperative (30\%) and 9 cases with worse BCVA postoperative (18 percent) and this confirms the positive effect of ranibizumab injection on BCVA.

From the studies done in post intravitreal ranibizumab injection; Guesspie et al. ${ }^{24}$ compared BCVA before and after ranibizumab injection on 18 eyes have reported a significant improvement in the postoperative BCVA.

The findings of our study compare favourably with this study and also with Park YU et al. ${ }^{19}$ who reported that the mean preoperative BCVA on snellen's was $(0.6 \pm 0.3)$ while the mean postoperative BVCA on snellen's was $0.7 \pm 0.3$ implying a good visual outcome after intravitreal injection of ranibizumab.
In addition to comparing the BCVA preoperative and postoperative we studied also the effect of the postoperative choroidal and retinal thickness on the postoperative BCVA, a statistical significance was existing $(p=0.016)$ negative correlation among the postoperative retinal thickness and the postoperative BCVA among the investigated patients with $(r=$ 0.338). Many previous papers supported this finding.

Even though a negative correlation $(r=-0.038)$ was existing among the postoperative $\mathrm{CT}$ and the postoperative BCVA among the studied cases but it was statistically insignificant correlation $(p=0.795)$. This result also agreed with previous studies as Glenn et $\mathrm{al}^{27}$ reported that central CT reduced after antiVEGF treatment for DME but it cannot be accompanied by any functional or anatomical consequences in eyes with DME as there's no significant correlation among the variation in CT and the variation in visual acuity.

In the current work we studied the effect of the postoperative choroidal and retinal thickness on the postoperative visual acuity but our primary target was to compare the consequences of ranibizumab as AntiVEGF on the CT by comparing the thickness before and after injection and our results as supported by many previously published papers confirmed that the subfoveal CT is significantly reduced after ranibizumab injection. These findings may be clarified as declared above by the outcome of ranibizumab on the choroidal vasculature. Along with the findings of this study, more experimental data on choroidal vasculature and more accurate means of choroidal measurement as well as a longer follow up period should be studied to provide better explanations for the CT variations in response to intravitreal injection of ranibizumab.

\section{CONCLUSION}

There was a significant change among the preoperative subfoveal CT and postoperative subfoveal CT. The higher baseline SFCT is usually associated with greater response after Anti-VEGF treatment and the reduction in CT following injection of ranibizumab reflect the effect of ranibizumab on the choroidal vasculature which needs additional investigation.

\section{REFERENCES}

1. Antonetti DA, Klein R, Gardner TW. Diabetic retinopathy. N Engl J Med 2012; 366:1227-1239 .

2. Bhagat N, Grigorian RA, Tutela A, Zarbin MA. Diabetic macular edema: pathogenesis and treatment. SurvOphthalmol 2009; 54:1-32 .

3. Spaide RF, Koizumi H, Pozzoni MC. Enhanced depth imaging spectral-domain optical coherence 
tomography. Am J Ophthalmol 2008; 146(4):496500 .

4. Cao J, McLeod S, Merges CA. Choriocapillaris degeneration and related pathologic changes in human diabetic eyes. Arch Ophthalmol 1998; 116(5): 589-597.

5. Nagaoka T, Kitaya N, Sugawara R. Alteration of choroidal circulation in the foveal region in patients with type 2 diabetes. $\mathrm{Br} J$ Ophthalmol 2004; 88(8):1060-3.

6. ManjunathV, TahaM, Fujimoto JG. Choroidal thickness in normal eyes measured using Cirrus HD optical coherence tomography. Am J Ophthalmol 2010; 150(3):325-9.

7. Panozzo G, Gusson E, Parolini B. Role of OCT in the diagnosis and follow-up of diabetic macular edema. Seminar Ophthalmology 2003; 18:74-81.

8. Schocket LS, Brucker AJ, Niknam RM. Foveolar choroidal hemodynamics in proliferative diabetic retinopathy. Interventive Ophthalmology 2004; 25:89-94.

9. Margolis R, Spaide RF. A pilot study of enhanced depth imaging optical coherence tomography of the choroid in normal eyes. American Journal of Ophthalmology 2009; 147:811-5.

10. Manjunath V, Taha M, Fujimoto JG. Choroidal thickness in normal eyes measured using Cirrus HD optical coherence tomography. Am J Ophthalmol 2010; 150:325-9.

11. Fujiwara $T$, Imamura $Y$, Margolis R. Enhanced depth imaging optical coherence tomography of the choroid in highly myopic eyes. American Journal of Ophthalmology 2009; 148:445-50.

12. Ikuno $\mathrm{Y}$, Kawaguchi $\mathrm{K}$, Nouchi T. Choroidal thickness in healthy Japanese subjects. Invest Ophthalmology Visual Science 2010; 51:2173-6.

13. Chen TC, Cense B, Miller JW. Histologic correlation of in vivo optical coherence tomography images of the human retina. American Journal of Ophthalmology 2006; 141:1165-8 .

14. Spaide RF, Koizumi H,Pozzoni MC. Enhanced depth imaging spectral domain optical coherence tomography. American journal of ophthalmology2008; 146:496-500.

15. Yiu GI, Pecen PA, Sarin NE. Characterization of the choroid-scleral junction and suprachoroidal layer in healthy individuals on enhanced-depth imaging optical coherence tomography. JAMA Ophthalmology 2014; 132(2):174-81.

16. Hee MR, Puliafito CA, Duker JS. Topography of diabetic macular edema with optical coherence tomography. Ophthalmology 1998; 105:360-70 .

17. Ikuno $Y$, Kawaguchi K, Nouchi $T$, Yasuno $Y$. Choroidal thickness in healthy Japanese subjects. Invest Ophthalmology Visual Science 2010; 51:2173-6.

18. Erkul SE, Kapran ZI, Uyar MO. Quantitative analysis of subfoveal choroidal thickness using enhanced depth imaging optical coherence tomography in normal eyes. International ophthalmology 2014; 34(2):35-40.

19. Park YU, Chung HY, Kim HY. Effects of Diabetic Retinopathy and Intravitreal Bevacizumab Injection on Choroidal Thickness in Diabetic Patients. Journal of Korean Ophthalmological Society 2013; 54(10):1520-5.

20. Lee SH, Kim JA, Chung HY. Changes of Choroidal Thickness after Treatment for Diabetic Retinopathy. Posted online on February 6, 2014. (Available http://www.ncbi.nlm.nih.gov/pubmed/24502275 accessed on 19th May 2014 at 2:07 pm(

21. Laíns IN, Figueira JO, Santos AN. Choroidal thickness in diabetic retinopathy: The Influence of Antiangiogenic Therapy. Retina 2014; 34(6):1199207.

22. Nadim Rayess, Ehsan Rahimy, Gui-shuang Ying. Baseline Choroidal Thickness as a Predictor for Response to Anti-Vascular Endothelial Growth Factor Therapy in Diabetic Macular Edema. Am J Ophthalmol 2015; 159(1): 85-91.

23. Kim JT, Lee DH, Joe SG. Changes in choroidal thickness in relation to the severity of retinopathy and macular edema in type 2 diabetic patients. Invest Ophthalmol. Vis Sci 2013; 54:3378-84 .

24. Querques G, Bux AV, Martinelli D, Iaculli C, Del Curatolo MV, Delle Noci N. Short-term fluctuation of diabetic macular edema after intravitreal ranibizumab injection. Retina. 2009 Oct;29(9):1274-81. doi: 10.1097/IAE.0b013e3181aa8e50. PMID: 19696699.

25. Diabetic Retinopathy Clinical Research Network, Browning DJ, Glassman AR, Aiello LP, Beck RW, Brown DM, Fong DS, Bressler NM, Danis RP, Kinyoun JL, Nguyen QD, Bhavsar AR, Gottlieb J, Pieramici DJ, Rauser ME, Apte RS, Lim JI, Miskala PH. Relationship between optical coherence tomography-measured central retinal thickness and visual acuity in diabetic macular edema. Ophthalmology. 2007 Mar;114(3):525-36. doi: 10.1016/j.ophtha.2006.06.052. Epub 2006 Nov 21. PMID: 17123615; PMCID: PMC2585542.

26. Keane PE, Alasil TA, Updike JA, Dustin LA, Ouyang LA, Walsh AL, Sadda SE. Relationship between optical coherence tomography retinal parameters and visual acuity in diabetic macular edema. Ophthalmology 2010; 117(12): 2379-86.

27. Yiu G, Manjunath V, Chiu SJ, Farsiu S, Mahmoud TH. Effect of anti-vascular endothelial growth factor therapy on choroidal thickness in diabetic macular edema. Am J Ophthalmol. 2014 Oct;158(4):745-751.e2. doi: 10.1016/j.ajo.2014.06.006. Epub 2014 Jun 19. PMID: 24952275; PMCID: PMC4443902. 